PAPER

\section{Fully automatic left ventricular myocardial strain estimation in 2D short-axis tagged magnetic resonance imaging}

To cite this article: Pedro Morais et al 2017 Phys. Med. Biol. 626899

View the article online for updates and enhancements.
Related content

- $\frac{\text { Regional assessment of LV wall in }}{\text { infarcted heart using tagged MRI and }}$
$\frac{\text { cardiac modelling }}{\text { Zeinab Jahanzad, Yih Miin Liew, Mehmet }}$
Bilgen et al.
- Automated segmentation and
$\frac{\text { reconstruction of patient-specific cardiac }}{\text { anatomy and pathology from in vivo MRI }}$
Jordan Ringenberg, Makarand Deo, Vijay
Devabhaktuni et al.
- The effect of myocardial segmentation
errors on flow estimates
J Biglands, D Magee, R Boyle et al.




\title{
Fully automatic left ventricular myocardial strain estimation in 2D short-axis tagged magnetic resonance imaging
}

\author{
Pedro Morais ${ }^{1,2,3,9,10}$, Sandro Queirós ${ }^{1,2,4,10}$, Brecht Heyde ${ }^{1}$, \\ Jan Engvall ${ }^{5,6,7}$, Jan D'hooge ${ }^{1}$ and João L Vilaça ${ }^{2,8}$ \\ ${ }^{1}$ Lab on Cardiovascular Imaging \& Dynamics, Department of Cardiovascular \\ Sciences, KULeuven-University of Leuven, Leuven, Belgium \\ 2 ICVS/3B's—PT Government Associate Laboratory, Braga/Guimarães, Portugal \\ ${ }^{3}$ Instituto de Ciência e Inovação em Engenharia Mecânica e Engenharia Industrial, \\ Faculdade de Engenharia, Universidade do Porto, Porto, Portugal \\ 4 Algoritmi Center, School of Engineering, University of Minho, Guimarães, Portugal \\ 5 Department of Clinical Physiology, Linköping University, Linköping, Sweden \\ ${ }^{6}$ Department of Medical and Health Sciences, Linköping University, Linköping, \\ Sweden \\ ${ }^{7}$ Center for Medical Image Science and Visualization (CMIV), Linköping University, \\ Linköping, Sweden \\ ${ }^{8}$ DIGARC—Polytechnic Institute of Cávado and Ave, Barcelos, Portugal \\ E-mail: pedromorais@med.uminho.pt
}

Received 6 March 2017, revised 1 July 2017

Accepted for publication 5 July 2017

Published 7 August 2017

\section{Abstract}

Cardiovascular diseases are among the leading causes of death and frequently result in local myocardial dysfunction. Among the numerous imaging modalities available to detect these dysfunctional regions, cardiac deformation imaging through tagged magnetic resonance imaging (t-MRI) has been an attractive approach. Nevertheless, fully automatic analysis of these data sets is still challenging. In this work, we present a fully automatic framework to estimate left ventricular myocardial deformation from t-MRI. This strategy performs automatic myocardial segmentation based on B-spline explicit active surfaces, which are initialized using an annular model. A non-rigid image-registration technique is then used to assess myocardial deformation. Three experiments were set up to validate the proposed framework using a clinical database of 75 patients. First, automatic segmentation accuracy was evaluated by comparing against manual delineations at one specific cardiac

\footnotetext{
${ }^{9}$ Life and Health Sciences Research Institute (ICVS), Universidade do Minho, Campus de Gualtar, 4710-057

Braga, Portugal.

${ }^{10}$ Joint first authorship. 
phase. The proposed solution showed an average perpendicular distance error of $2.35 \pm 1.21 \mathrm{~mm}$ and $2.27 \pm 1.02 \mathrm{~mm}$ for the endo- and epicardium, respectively. Second, starting from either manual or automatic segmentation, myocardial tracking was performed and the resulting strain curves were compared. It is shown that the automatic segmentation adds negligible differences during the strain-estimation stage, corroborating its accuracy. Finally, segmental strain was compared with scar tissue extent determined by delay-enhanced MRI. The results proved that both strain components were able to distinguish between normal and infarct regions. Overall, the proposed framework was shown to be accurate, robust, and attractive for clinical practice, as it overcomes several limitations of a manual analysis.

Keywords: tagged magnetic resonance imaging, fully automatic segmentation, non-rigid image registration, strain estimation

S Supplementary material for this article is available online

(Some figures may appear in colour only in the online journal)

\section{Introduction}

According to the 2015 cardiovascular disease (CVD) epidemiological report, CVDs are the leading cause of death in Europe, being responsible for $45 \%$ of all deaths (Townsend et al 2015). Since several CVDs manifest as local myocardial dysfunction, the assessment of regional myocardial deformation has gained considerable attention (Hui and Amini 2012, Smiseth et al 2015).

Tagged magnetic resonance imaging (t-MRI) is an imaging technique that induces a pattern in the tissue of interest by spatially presaturating the tissue magnetization (Axel et al 2005, Hui and Amini 2012). These patterns are called tags and work as landmarks, which move/ deform along with the myocardium throughout the cardiac cycle, therefore easing the assessment of local deformation as they visually enhance certain motion components (e.g. torsion). Nevertheless, the analysis of these data sets remains challenging due to tag fading, the low spatial resolution, and the high variability between different data sets, ultimately hampering the identification and assessment of the underlying cardiac anatomy (Axel et al 2005, Hui and Amini 2012, Chitiboi and Axel 2017).

Several solutions have been proposed to compute myocardial deformation from t-MRI images. A review of these methods is presented in Hui and Amini (2012) and Chitiboi and Axel (2017). All these methods exploit the spatial or frequency information contained within the tags to estimate the left ventricle (LV) deformation throughout the cardiac cycle. While the tracking itself is commonly fully automatic (Osman et al 1999, Liu and Prince 2010, Miller et al 2013, Del-Canto et al 2015), its initialization is typically not. Indeed, myocardial segmentation (i.e. delineation of the target region) is essential, but it remains an either manual or semi-automatic process, thereby preventing myocardial strain estimation from being fully automatic. This interactive process is not only time-consuming and tedious, but also results in relatively high intra- and inter-observer measurement variability (Queirós et al 2014). In t-MRI images, the variability in contour definition tends to be worse than the remaining MRI sequences due to the heterogeneous nature of these images combined with tag fading over the cardiac cycle. As a result, images tend to be fairly noisy with myocardial boundaries being obscured or corrupted by tagging patterns (Qian et al 2005). To mitigate these issues, some 
authors have proposed different methodologies to segment the LV in t-MRI, the majority of which use a two-step strategy: an initial detagging technique to suppress the tags and partially recover the original information from the myocardial wall, followed by an image-segmentation strategy (Qian 2008, Shi et al 2012). Nevertheless, the detagged images typically have low contrast and are dependent on the signal-to-noise ratio (SNR) of the original image, hampering the correct segmentation of the cardiac wall (Qian 2008). Moreover, these techniques are typically not combined with a tracking methodology. As such, it remains difficult to verify whether they improve the assessment of regional cardiac function.

In the current work, a fully automatic strategy to estimate myocardial strain from t-MRI images is proposed. Its accuracy is computed in a large clinical database and is assessed against the traditional workflow, which relies on manual contouring followed by image tracking.

The current work introduces three novelties: (1) a robust LV detection and contour initialization methodology based on a signed annular shape identification strategy; (2) a fully automatic framework to accurately and efficiently estimate strain curves from t-MRI images based on an automatic segmentation method and a state-of-the-art image tracking approach; and (3) a validation of the proposed framework against the traditional workflow using a multicenter clinical database.

\section{Materials and methods}

\subsection{Data description}

A clinical database of patients with suspicion of ongoing (chronic) myocardial ischemic heart disease, obtained from a multi-center imaging trial was used (DOPPLER-CIP, determining optimal non-invasive parameters for the prediction of left ventricular morphological and functional remodeling in chronic ischemic patients (Rademakers et al 2013)). A total of 75 data sets were acquired by two independent centers in Europe, namely 34 cases from KUL (KU Leuven, Belgium) and 41 cases from LIO (Linköping University, Sweden). Each data set consists of a short-axis view at the base, mid-ventricular, and apical slice. For KUL, the images were acquired with a $1.5 \mathrm{~T}$ Ingenia scanner (Philips HealthCare, the Netherlands) with a tag orientation of $0_{\circ}$, resolution of $1.37 \times 1.37 \mathrm{~mm}$, output image resolution of $256 \times 256$ pixels, slice thickness of $8 \mathrm{~mm}$, repetition time of $3.0 \mathrm{~mm}$, echo time of $4 \mathrm{~ms}$, flip angle of $13^{\circ}$, and a total number of frames that varies between 20 and 25 was used. The images of LIO were obtained using a 1.5 Achieva scanner (Philips Healthcare, the Netherlands) with a tag orientation of $45^{\circ}$, resolution of $1.25 \times 1.25 \mathrm{~mm}$, output image resolution of $256 \times 256$ pixels, and a slice thickness of $6 \mathrm{~mm}$, repetition time of $4.48 \mathrm{~mm}$, echo time of $4.48 \mathrm{~ms}$, flip angle of $15^{\circ}$, and a total number of 15-20 phases. For both centers, the spatial modulation of the magnetization technique was used to create the tags.

\subsection{Framework overview}

The workflow of the fully automatic methodology is shown in figure 1. It starts with a fastmyocardial wall segmentation technique based on the B-spline explicit active surfaces (BEAS) framework (Barbosa et al 2012), followed by an image-registration technique to estimate the LV deformation (Morais et al 2013).

The segmentation stage identifies the myocardial region in one specific cardiac phase, generating 2D contours of its inner (i.e. endocardium) and outer (i.e. epicardium) borders (section 2.3). The contours are estimated per level, and they delimit the boundaries of the target anatomy inside the full image. Subsequently, these contours are propagated throughout the 


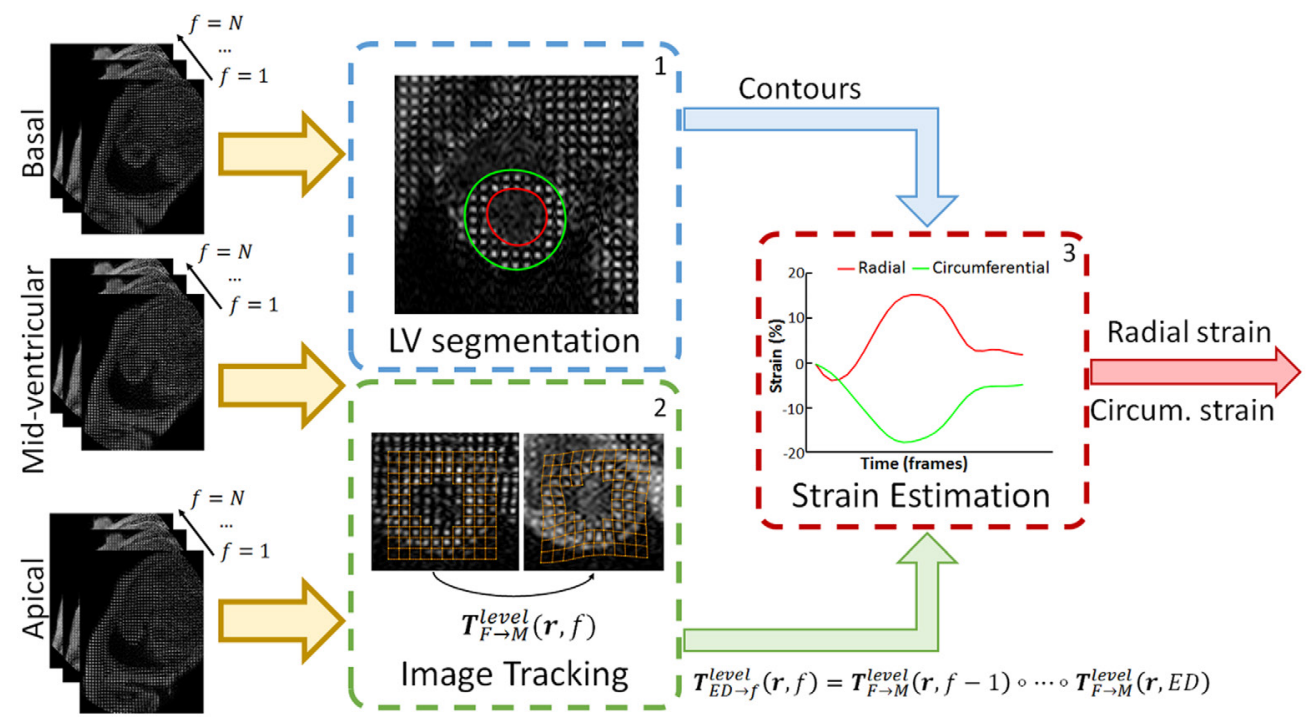

Figure 1. Overview of the proposed fully automatic framework. Clinical parameters are estimated as the result of three conceptual blocks: (1) identification and segmentation of the left ventricular wall; (2) tracking of the myocardial wall throughout the cardiac cycle; and (3) computation of local radial and circumferential (circum.) strain curves.

cardiac cycle using an image-registration approach (section 2.4.1). Specifically, the imageregistration method identifies the optimal transformation field between consecutive images, applying it to the initial contours and deforming them according to the estimated anatomical motion. Note that not only the myocardial borders are propagated but the full myocardial muscle is transformed, providing local information about its contraction and motion. The obtained myocardial motion field is then used to estimate local strain in the radial and circumferential directions (section 2.4.2).

\subsection{Fully automatic segmentation of the $L V$}

The automatic segmentation process relies on two stages (figure 2): (1) rough LV detection and region-of-interest (ROI) definition at the basal level; and (2) myocardial contour initialization and segmentation. Given that a t-MRI acquisition typically consists of three slices spaced relatively far apart (basal, mid-ventricular, and apical), 2D segmentations are sequentially applied from the basal to apical levels. Due to the typically higher image quality found at the basal slice, the LV detection stage is performed at this level only, being used to crop the original image and to initialize the subsequent segmentation stage.

During the LV detection stage (see section 2.3.1, figure 2(A)), an image-based dispersion filter is applied to enhance the tags and then binarize them. Subsequently, a novel signed annular shape identification strategy, referred to as signed annular Hough transform (SAHT), is applied to estimate the epicardial position and define an LV ROI.

In the second stage (see section 2.3.2, figure 2(B)), starting from the obtained LV ROI, a myocardial contour segmentation strategy is applied on a cropped t-MRI image. In contrast to the first LV detection stage, tag enhancement is now performed in the spectral domain. While multiple tag frequency components are found in the spectrum of the original uncropped image (due to the large field-of-view and the presence of multiple tags), the spectrum of the cropped 


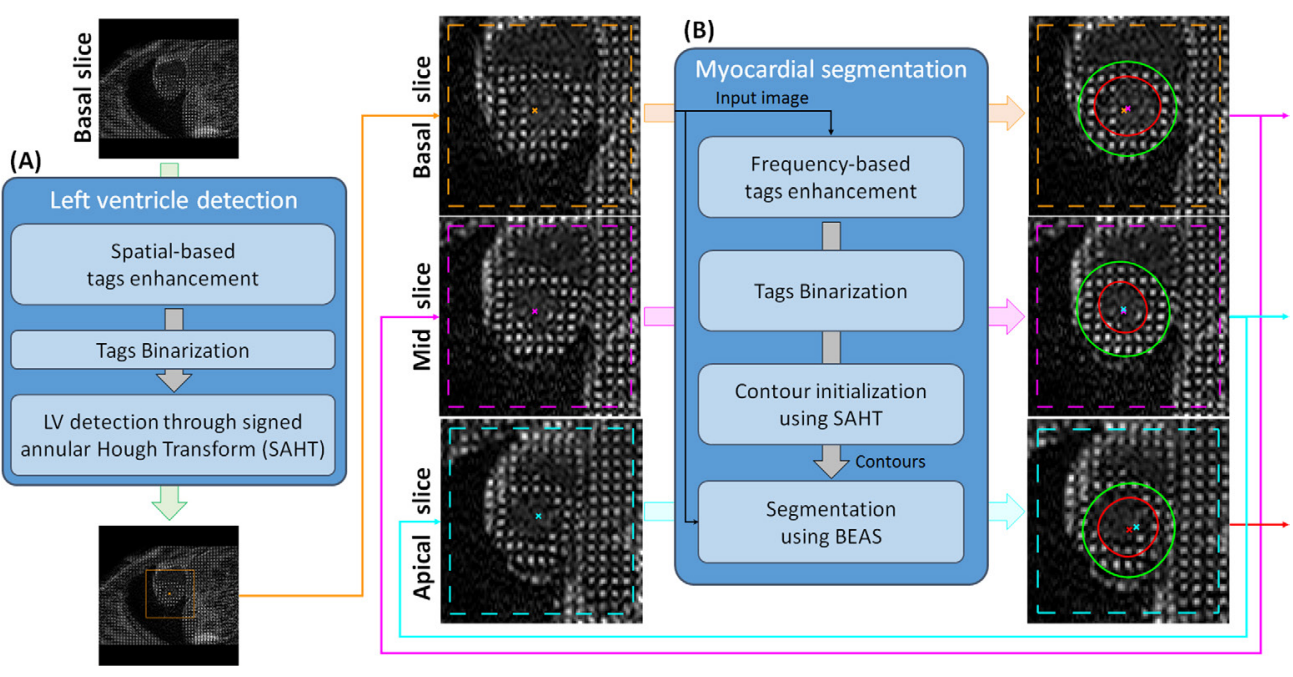

Figure 2. Schematic of the proposed automatic myocardial segmentation in tagged MRI.

version (i.e. after LV ROI detection) presents a clear definition of the myocardial tag frequency components, ultimately improving their detection accuracy. Next, these tag positions are again binarized and the myocardial contour initialized (endo- and epicardium) through the abovementioned SAHT method. The contours are then refined to the real anatomy using BEAS (Barbosa et al 2012). In contrast to the state-of-the-art methods working with detagged images, this step is applied on the original gray-scale image, thereby keeping the high contrast between the myocardial wall and the blood pool, while making the method less dependent on the image's SNR.

2.3.1. $L V$ detection. One of the major difficulties to automatically process MRI data sets is the correct identification of the LV. The acquired images show multiple organs besides the relevant cardiac chamber, complicating its identification. Particularly for t-MRI data sets, this tends to be challenging, both due to the presence of the tags on the entire image (including the blood pool) at the beginning of the sequence and due to tag fading.

As such, an automatic segmentation strategy cannot usually be applied at the end-diastolic (ED) frame. In this sense, a mid-systolic cardiac phase was used instead. It might be noted that although some authors previously proposed to use the final frame of the sequence to perform segmentation (Chandrashekara et al 2004), assuming therefore that the initial and last frames are taken at the same phase of the cardiac cycle, full cardiac cycle sequences are typically not acquired in clinical t-MRI data sets and usually present a low-image contrast at the final frame of the sequence.

2.3.1.1. Image-based tag enhancement and binarization techniques. Since the tags fade quickly inside the blood pool (in the first frames) and are then only superimposed on the tissue regions at the mid-diastolic frame used, the correct identification of these tags can be used to roughly detect the different shapes in the upper chest. For this, we combine both tag enhancement and binarization strategies.

The first step relies on a standard deviation filter based on local intensity, thus enhancing the heterogeneous image regions (i.e. the tag transitions). This filter defines the value of each 


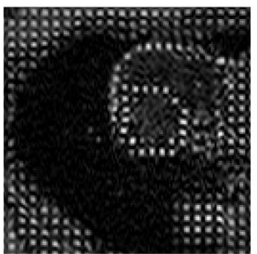

(a)

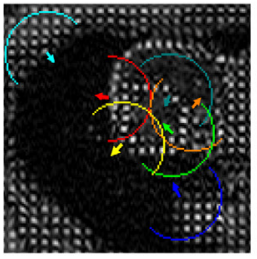

(f)

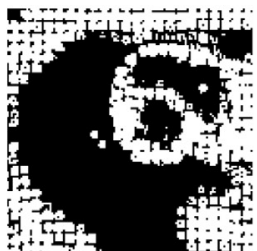

(b)

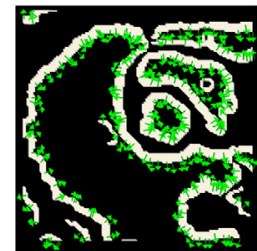

(c)

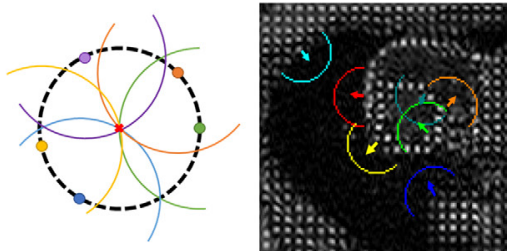

(d) (e)

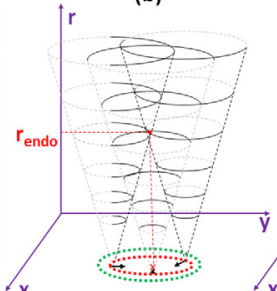

(g)

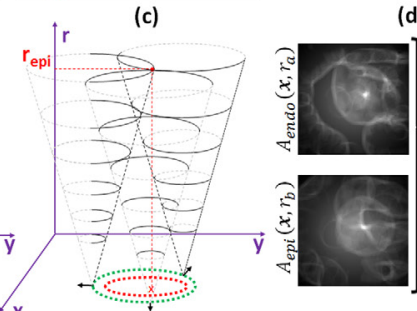

(h)

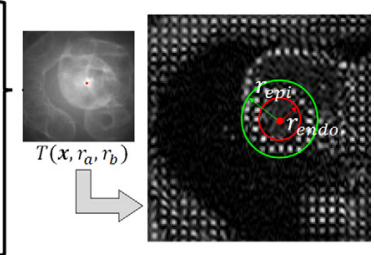

Figure 3. Voting process of the SAHT technique for LV detection. (a) Original example basal slice. (b) Binarized tag image obtained from (a). (c) Edge map of (b), and corresponding gradient vectors (green arrows). (d) Schematic of SAHT voting strategy. (e) and (f) Example semi-circles virtually generated from each edge point in (c), according to the edge gradient. Note that in (e), the semi-circles are oriented with the edge gradient (i.e. endocardial Hough), while in (f) they are along the opposite direction (i.e. epicardial Hough). (g) and (h) Illustrate the multiple radii voting strategy into a 3D accumulator. (i) Accumulator maps for a given radius value for both endo- and epicardial Hough, respectively, and final optimal center position (red circle) and endo/ epi radii (red and green arrow).

image pixel as the local intensity dispersion (measured through the standard deviation) of its neighborhood. Therefore, the filter size should be equal to the tag spacing (specifically, a kernel size of $\sim 4 \mathrm{~mm}$ was used as the inter-tag distance was $\sim 8 \mathrm{~mm}$ in the clinical database), consequently allowing the enhancement of the tag transitions, while maintaining the remaining regions. In the second step, a simple Otsu thresholding technique is used (Otsu 1975), which allows the separation of the image tags from the background. An example image after Otsu thresholding is shown in figure 3(b). Finally, we compute the edge map of this image according to Barbosa et al (2013), as shown in figure 3(c). Note that the method in Barbosa et al (2013) is a variant of an edge-detection algorithm providing the edge location and associated vertical and horizontal gradients. The gradient positions are defined on a 2D map. Both the edge map and gradients will make the identification of the LV easier, as explained in the next section.

2.3.1.2. SAHT. Some authors have previously presented strategies to identify the myocardium on cine-MRI data sets through signed annular models (Ciofolo et al 2008, Queirós et al 2013). These methods are based on the grayscale values and intensity transitions, assuming that the myocardium can be described as a dark object compared to both blood pools. Thus, multiple dark rings are searched on the image through block matching (Ciofolo et al 2008, Queirós et al 2013). However, due to the presence of multiple tag transitions, these strategies are not suitable for t-MRI images.

As such, we propose a novel signed annular model based on oriented Hough transforms. Instead of an exhaustive model identification on the grayscale image as customary in cineMRI, the SAHT method identifies the ROI using a voting strategy applied on the edge map obtained in the previous section, consisting of the following steps: 
(i) Multiple annular models with different radii are generated. Each model relies on two independent Hough transforms, one focusing on the identification of the endocardial region, termed endocardial Hough (figure 3(e)), and another targeting the epicardium, designated epicardial Hough (figure 3(f)). Moreover, in order to increase the robustness of the technique for LV detection, a signed version of the method is proposed. Thus, discretized semi-circles $\left(C_{m, k}\right)$ oriented based on the image gradient, $\varphi_{m}$, at each edge point, $m$, are generated through:

$$
\begin{gathered}
C_{m, k}\left(\varphi_{m}, r\right)=i\left(r \cos \left(\theta_{k}(p)\right)+x_{m}\right)+j\left(r \sin \left(\theta_{k}(p)\right)+y_{m}\right), \\
p=1,2, \ldots, N_{C} \quad \theta_{k}(p) \in\left\{\begin{array}{lr}
{\left[\varphi_{m}-\frac{\pi}{2}, \varphi_{m}+\frac{\pi}{2}\right],} & \text { if } k=\text { endo } \\
{\left[\varphi_{m}+\frac{\pi}{2}, \varphi_{m}+\frac{3 \pi}{2}\right],} & \text { if } k=\text { epi }
\end{array}\right.
\end{gathered}
$$

where $r$ is the radius of the semi-circle, $N_{C}$ is the number of discretized points used to represent each semi-circle, $k$ indicates which Hough transform is being applied (endo- or epicardium), $\left[x_{m}, y_{m}\right]$ are the edge positions, and $i j$ are the coordinates of the image domain.

(ii) Each semi-circle $C_{m, k}$ is centered at each point $m$ of the edge map, and the $\theta_{k}(p)$ value selected based on the expected myocardial intensity transition profile seen from the LV center position towards the myocardium. A white-to-black transition and a blackto-white transition is used for the endocardium $(k=$ endo) and epicardium $(k=$ epi, figures 3(a)-(c)), respectively. In this sense, at the real edges of the endocardium, the resulting endocardial Hough generates semi-circles that-combined-will vote for the center of the LV cavity (dark and light green semi-circles in figure 3(e)), while the epicardial Hough generates a scattered map (dark and light green semi-circles in figure 3(f)). In contrast, at the epicardium's real edges, the endocardial Hough creates a scattered map for the right ventricle (yellow, red, and blue semi-circles in figure 3(e)), while the epicardial Hough votes for the LV cavity center (yellow, red, and blue semi-circles in figure 3(f)). Note that this implementation is the inverse operation of testing multiple semi-circles positioned in multiple candidate centroids, which would have a very large number of possibilities to test (the two coordinates of the centroid and the radii for each possible centroid). Indeed, assuming a given circle (position $[x, y]$ and radius $r$ ), if we generate semi-circles centered in the points along its circumference with radius $r$, all semi-circles will intersect, i.e. vote, in the original centroid (i.e. $[x, y]$ ). This is the principle behind our SAHT approach (figure 3(d)).

(iii) Two independent accumulators $A_{k}$ are used to count the intersection points, each representing either the endo- or epicardial Hough transform. Let $\boldsymbol{x}$ represent each coordinate pair $[x, y]$ of the image, $A_{k}(\boldsymbol{x}, r)$ forms a $3 \mathrm{D}$ accumulator map with the third dimension representing the different tested models, i.e. different radii $r$ (figures $3(\mathrm{~g})$ and $(\mathrm{h})$ ). Since the t-MRI image acquisition is typically centered on the LV, a weighted voting strategy is used to further enhance the central positions of the accumulator map, penalizing the remaining regions and reducing the noise obtained on the map. As such, the voting process is performed using a confidence measure given by the distance to the center image position $\left(S_{\mathrm{I}}\right)$, through:

$$
A_{k}(x, r)=\sum_{m=1}^{N_{\text {Edge }}}\left\{\begin{array}{cl}
\left(1-\frac{x-S_{\mathrm{I}}^{2}}{D_{\max }}\right) & \text { if } C_{m, k}\left(\varphi_{m}, r\right) \bigcap x \neq \varnothing \\
0 & \text { if } C_{m, k}\left(\varphi_{m}, r\right) \bigcap x=\varnothing
\end{array}\right.
$$



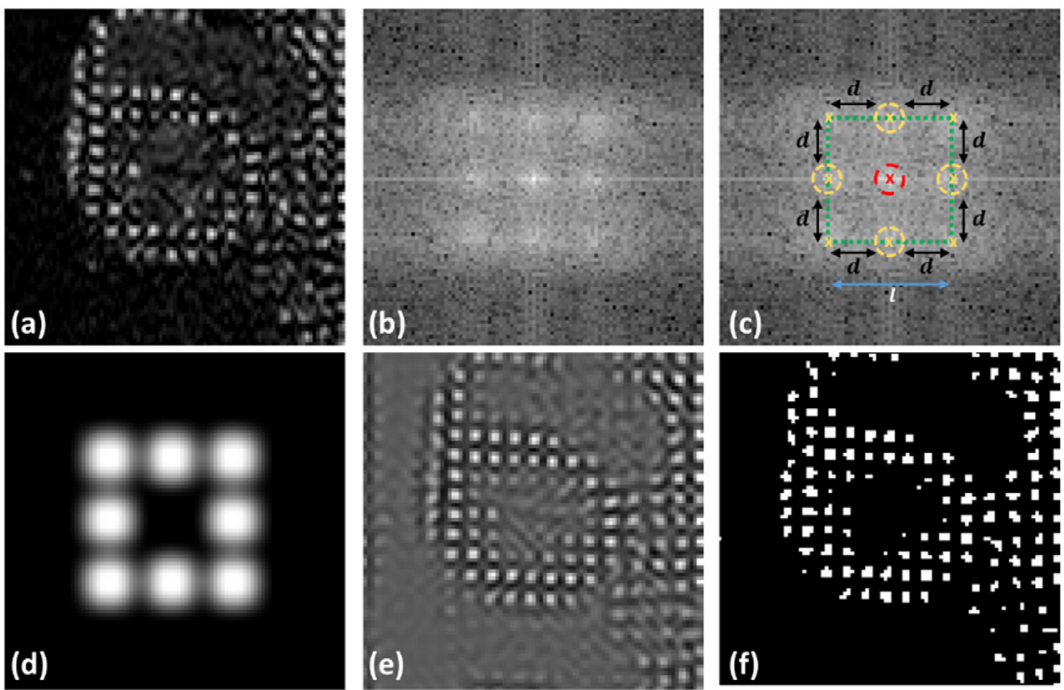

Figure 4. Frequency-based tag enhancement and binarization technique. (a) Original image, (b) spectral representation of (a), (c) identification of the eight maximum tag frequencies (yellow crosses - tags frequencies, red cross-zero frequency component) and square fitting with size $l$ using the distance $d$ between consecutives candidates, (d) resulting tag enhancement filter, (e) tag enhanced image and (f) binarization of (e) through Otsu thresholding.

where $N_{\text {Edge }}$ is the total number of edge points, and $D_{\max }$ the diagonal length of the cropped image.

(iv) The endo- and epicardial accumulators are combined into one fused accumulator $(T)$ :

$$
\begin{gathered}
T\left(\boldsymbol{x}, r_{a}, r_{b}\right)=A_{\text {endo }}\left(\boldsymbol{x}, r_{a}\right)+A_{\mathrm{epi}}\left(\boldsymbol{x}, r_{b}\right), \\
a \in\left[1, R_{\max }-d_{\mathrm{w}}\right] \text { and } b \in\left[a+d_{\mathrm{w}}, R_{\max }\right],
\end{gathered}
$$

where $R_{\max }$ is the maximum radius used and $d_{\mathrm{w}}$ represents the minimal wall thickness. Note that the $d_{\mathrm{w}}$ term prevents overlaps between contours. Since both accumulators are combined into a fused one, only one center position is found, making it robust to local minima (figures $3(\mathrm{~g})$ and $(\mathrm{h})$ ).

(v) The maximum in $T$ identifies the optimal center position of the annular model, while the associated radii indicate the optimal endo- and epicardial radii of the annular model (figure 3(i)). Furthermore, the epicardial contour is used to define a ROI, and crop the original t-MRI image, as shown in figure 4(a). An overview of its implementation is given in multimedia material I (stacks.iop.org/PMB/62/6899/mmedia).

\subsubsection{Myocardial segmentation.}

2.3.2.1. Frequency-based tag enhancement and binarization technique. Starting from the cropped grayscale image (figure 4(a)), the spectrum is computed using the traditional 2D fast Fourier transform (FFT) technique. Since the tags are generated using radio-frequency pulses applied during the acquisition, a specific representation of the tags on the Fourier domain is observed (figure 4(b)), specifically a central peak defining the zero-frequency component and eight secondary peaks representing the first harmonic of the tag frequencies (Qian 2008). In 
this sense, and in contrast to section 2.3.1, an accurate estimation of the tag positions can now be obtained.

In order to easily identify the frequency components associated with the tags, the zerofrequency component was removed through an ideal high-pass filter with cutoff frequency at a 10 pixel radius (red circle in figure 4(c)). Since a similar representation of the tag frequencies on the spectrum is usually observed for different t-MRI data sets, a fixed cutoff frequency was empirically defined. Then, candidates of the first harmonic of the tag frequencies are detected as the eight locations with highest intensity (yellow crosses in figure 4(c)). A distance larger than 10 pixels must exist between candidates to avoid multiple detections from the same component. Subsequently, and in order to increase the detection robustness, the average distance $d$ between consecutive candidates is used to define the size $l$ of the square that represents the first harmonic of the tag frequencies (green square in figure 4(c)). This technique is feasible for both $0^{\circ}$ and $45^{\circ}$ tag grids.

Based on the final eight candidate locations, a morphological dilation with a disk of 7 pixel $(\sim 10 \mathrm{~mm})$ radii is applied and the result is then convolved with a Gaussian filter (sigma of $\sim 4 \mathrm{~mm}$ ) to prevent artifacts. The resulting filter (figure $4(\mathrm{~d})$ ) is multiplied with the original spectrum, and the inverse FFT technique is used to reconstruct a tag-enhanced image (figure 4(e)). Subsequently, the tags are binarized using Otsu thresholding (Otsu 1975) (figure 4(f)).

Finally, the frequency-based enhanced and binarized image is used to accurately initialize the endo- and epicardial contour through the abovementioned SAHT strategy.

It should be noted that the proposed frequency-based filter could not be applied on the original (non-cropped) image. Given that we work with an image at mid-systole, and given that different areas outside the myocardium will have faded at different rates, multiple (erroneous) tag components would be retained throughout spectral filtering, hampering the correct identification of the myocardial wall.

2.3.2.2. Segmentation using BEAS. Recently, the BEAS framework proved to be suitable for the segmentation of multiple cardiac structures acquired with different imaging modalities (Queirós et al 2014, 2016). A clinical validation (Queirós et al 2015) of the method showed its high feasibility, high accuracy, and low computational time, being attractive for clinical practice.

In this work, we propose to use BEAS to accurately delineate the LV on t-MRI images. The method is applied on the cropped grayscale image. Note that, in contrast to previous approaches with detagged images, this framework uses the original t-MRI image, thereby keeping the high contrast between myocardium and blood pool.

Segmentation of both endo- and epicardial boundaries was performed simultaneously using a coupling strategy (Queirós et al 2013). More specifically, both are modeled as a combination of two explicit functions representing the myocardial shape and thickness, respectively. While the first function has the ability to recover complex myocardial shapes, the second provides local adaptation of the contours, being possible to apply smoothing factors independently for each. In this sense, by applying high factors to the local wall thickness, irregular LV shapes caused by the tags' block-wise appearance are prevented, without affecting the algorithm's overall ability to capture complex and irregular myocardial shapes (e.g. pathological anatomies).

A local energy term (local Chan-Vese) was used to search for the optimal position between two homogeneous regions, while a global energy term assured that the whole myocardial wall has a homogeneous intensity by penalizing any region that deviates from such an assumption. Their combination allows local refinement of the segmented contour, while taking into account 
that a global coherent annular structure (i.e. the myocardium) should be found. Further details on the segmentation strategy and parameters can be found in Queirós et al (2013).

Finally, the obtained contour is used to initialize the segmentation in the remaining levels. Specifically, the contour obtained in one level (e.g. basal slice) is used to define a ROI to crop the subsequent level (e.g. mid-ventricular slice). This ROI is used as a starting point to the segmentation strategy (section 2.3.2).

\subsection{Motion estimation and strain computation}

2.4.1. Motion tracking. In order to track the t-MRI images throughout the cardiac cycle, a non-rigid image-registration technique was applied. Previous studies have shown that this method is attractive when compared with other alternatives (Smal et al 2011). Traditionally, registration is performed in a pair-wise fashion between subsequent frames. However, for t-MRI, suboptimal results tend to be obtained due to tag fading, the noisy image quality, and the tag flushing inside the blood pool (Morais et al 2013).

Instead, a sequential 2D $+\mathrm{t}$ transformation model was proposed in Morais et al (2013). This model computes motion in a temporal coherent framework taking all images into account at once. It can better penalize non-smooth motion caused by tag fading or flushing, while maintaining enough flexibility to extract physiological deformation. The method was previously tested on synthetic and clinical data sets, proving its high accuracy and ability to detect dysfunctional regions (Morais et al 2013).

2.4.2. Strain estimation. Strain curves are computed using the technique described in Morais et al (2013). In this case, the regional deformation of the myocardial wall is assessed by a set of points positioned within the myocardial wall. In each segment, five and ten points are used in the radial and circumferential directions, respectively. Segments are defined according to the model described in Cerqueira et al (2002). The displacement of all sampled points relative to their initial position is then found by consecutively cumulating the transformation fields obtained through the tracking technique. As such, strain $\left(\varepsilon_{N}\right)$ is computed relative to the enddiastolic frame, as described in:

$$
\varepsilon_{N}(f)=\frac{D_{N}(f)-D_{N}(E D)}{D_{N}(E D)},
$$

with $D_{N}(f)$ representing the distance between two adjacent points in either the radial or circumferential direction at frame $f$, and similarly $D_{N}(\mathrm{ED})$ being the respective distances at ED.

\subsection{Implementation details}

Due to the presence of tags in the blood pool at the initial frames, we empirically decided to apply the LV segmentation strategy at the 4th frame. Since the cardiac strain is usually measured with respect to the ED frame, a 'reverse' propagation of the myocardial contour between the 4th frame and ED was performed using the proposed tracking technique. Then, the tracking technique was applied again, but now to the entire sequence and strain curves computed.

The LV detection and contour initialization with the SAHT method used annular models with radii between 15 and $40 \mathrm{~mm}$, representing the expected LV wall thickness. Two independent number of clusters were used in the binarization, namely two and three levels for the LV detection and myocardial segmentation stage, respectively, with the cluster with maximum intensity 
being kept. A tolerance of $25 \mathrm{~mm}$ for each side of the detected ROI was given to compensate for possible slice misalignment and sub-optimal detections. All parameters were experimentally selected to achieve a stable performance for all the patients. Further details on parameter selection can be found in multimedia material II (stacks.iop.org/PMB/62/6899/mmedia).

\subsection{Experiments}

Three experiments were set up to validate the proposed framework, namely: (1) automatic segmentation performance; (2) sensitivity of the strain values with respect to automatic or manual contouring; and (3) clinical validation through the comparison of strain values obtained from t-MRI data sets in myocardial segments with or without scar tissue.

2.6.1. Automatic-segmentation performance. Each dataset was manually segmented by two observers. The segmentation was performed for each short-axis view at mid-systole (4th frame). The slices in which one of the observers could not identify the myocardium were excluded ( 21 of 225 slices). By averaging the contours of both observers, reference segmentations were obtained.

Next, these data sets were processed by the proposed segmentation technique. Differences between the automatic and mean contour of the two observers were analyzed at the 4th frame in terms of average perpendicular distance (APD), the Dice coefficient, and the Hausdorff distance (i.e. maximum APD). Each result was compared to the inter-observer variability (IOV, defined as the differences between both observers' manual analysis) using a Wilcoxon matched-pairs signed rank test $(p<0.05)$. Moreover, to verify if the performance was different for each ventricular level, a Mann- Whitney test $(p<0.05)$ was performed. The segmentation was considered to be successful when the APD remained under $5 \mathrm{~mm}$. Note that this threshold is also used when evaluating cine-MRI segmentation algorithms (Queirós et al 2014).

The accuracy of each stage of the proposed strategy was also assessed by comparing the center of mass of the automatic and manual contours. A Mann-Whitney test $(p<0.05)$ was used to assess statistical differences.

Finally, the computational time of the automatic framework was registered using MATLAB code (version 2015a) running on an Intel (R) i7 CPU at $2.8 \mathrm{GHz}, 16 \mathrm{~GB}$ of RAM and a Microsoft Windows(R) 8 operating system.

2.6.2. Strain-estimation performance. In order to assess the sensitivity of the tracking approach to the underlying segmentation methodology, the end-systolic (ES) strain values and global strain curves obtained with both segmentation approaches were contrasted. The ES was identified by an expert. Only cases in which such annotation was available were included in this analysis, resulting in 175 slices (64 basal, 61 mid-ventricular, and 50 apical levels out of the original 204 slices).

Global and segmental strain values were compared by computing a linear correlation coefficient and Bland-Altman (BA) analysis, expressed as the bias (mean difference) and limits of agreement (LOA, 1.96 times the standard deviation). At ES, performance was compared per level and per segment. A two-tailed paired $t$-test and $F$-test were used to identify statistically significant differences $(p<0.05)$ in the obtained biases and LOAs, respectively. Note that, for segmental analysis, the inferior right ventricular insertion point was manually defined by one observer, which was then used to define the 16 segments for both automatic and manual approaches. In this sense, only the contours differences' influence on the strain estimation 
is retained. The difference between the full strain curves was assessed using their cross-correlation coefficient. A two-tailed paired $t$-test against zero $(p<0.05)$, was used to identify statistically significant biases. Finally, the IOV was computed by tracking the contours of the individual observers rather than starting from the average contours.

2.6.3. Strain estimated from t-MRI in myocardial segments with different amount of scarring. Strain estimated from t-MRI (computed through the fully automatic approach) was contrasted between myocardial segments with a different amount of scar tissue obtained from delay-enhancement (DE) MRI data sets of the same patient. This study was performed on the abovementioned 175 slices. One expert computed the percentage of scar tissue per segment. Using the strategy previously described in Ypenburg et al (2007), to each segment was assigned a score describing the extent of the scarring: 0 - absence of scarring; 1 -scar tissue with extent $1 \%-25 \% ; 2$ - extent $26 \%-50 \%$; 3-extent $51 \%-75 \%$ and 4 - extent $76 \%-100 \%$. Note that the segments were defined using the 16 segments model. A score of 1 and 2 typically indicates a non-transmural infarct segment, whereas 3 and 4 point to an infarct extending transmurally. The myocardial value obtained in each segment was thus divided in three classes (normal, non-transmural, and transmural) based on the scar score, and finally statistically compared through an unpaired $t$-test $(p<0.05)$.

\section{Results}

\subsection{Automatic-segmentation performance}

A total of $58(77 \%)$ apical, 71 (95\%) mid-ventricular, and $75(100 \%)$ basal slices were analyzed by the observers (total of 204 slices). The automatic strategy was able to successfully identify the LV in all cases, i.e. the center of the annular model was estimated inside the LV. The subsequent segmentation was successful in 97\% (197 slices) and 99\% (202 slices) for the endo- and epicardium respectively, showing the robustness of the technique even in clinical data sets with different acquisition parameters. The mean computational time for each data set (i.e. 3 levels) was approximately $180 \mathrm{~s}$ for each observer versus $1.17 \pm 0.08 \mathrm{~s}$ for the automatic approach.

Figure 5 shows the error of the LV center during the three stages of the segmentation technique. A significant reduction of the centroid error between the initial ROI and the initialization step was always observed, while the remaining steps showed a similar error. Table 1 lists the automatic segment error per level, the global error, and the IOV. Generally, the error of the automatic approach was similar to the IOV, particularly in the endocardium.

Finally, representative segmentation examples at mid-systole are shown in figure 6 at different levels.

\subsection{Strain-estimation performance}

Table 2 shows the global and segmental strain results in terms of linear regression and BA analysis. Although wider LOAs were found in the segmental analysis compared to the global strain, these differences in terms of segmental strain were not statistically significantly different from those that were obtained starting from the manual references. In contrast, and in agreement with the results from the global analysis, a statistically significant bias existed between the automatic technique and the IOV for the radial strain only. Figure 7 shows a tracking example and the obtained strain curves, where a similar result was obtained by both 


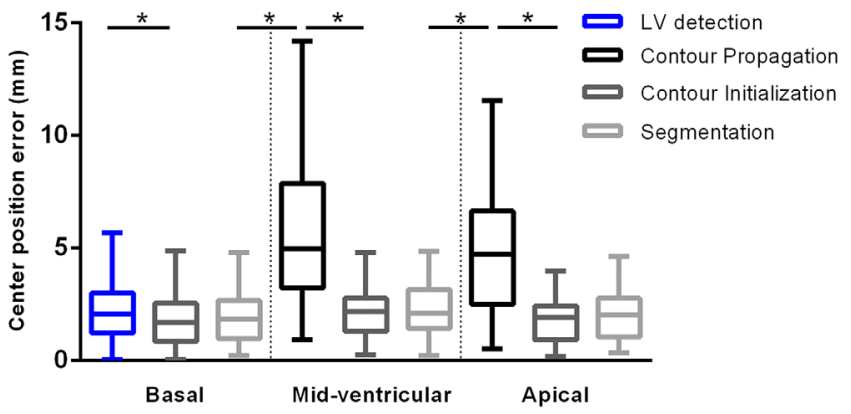

Figure 5. Center position error throughout the different steps of the automatic segmentation approach. In each step, the estimated center position is compared with the center of mass of the manual reference. $* p<0.05$, Mann-Whitney test between consecutive steps of the method.

Table 1. Segmentation errors between automatic and manual contours.

\begin{tabular}{lllllllll}
\hline & \multicolumn{2}{c}{ APD $(\mathrm{mm})$} & & \multicolumn{2}{c}{ DICE } & & \multicolumn{2}{c}{ Hausdorff $(\mathrm{mm})$} \\
\cline { 2 - 3 } & Endo & Epi & & Endo & Epi & & Endo & Epi \\
\hline Basal & $2.44 \pm 1.34^{\mathrm{b}}$ & $2.14 \pm 0.82^{\mathrm{b}}$ & & $0.89 \pm 0.06^{\mathrm{b}}$ & $0.94 \pm 0.02^{\mathrm{b}}$ & & $5.28 \pm 2.41$ & $4.86 \pm 0.55^{\mathrm{b}}$ \\
Mid & $2.56 \pm 1.23$ & $2.38 \pm 1.21^{\mathrm{b}}$ & & $0.89 \pm 0.06$ & $0.93 \pm 0.03^{\mathrm{b}}$ & & $5.43 \pm 2.16$ & $5.14 \pm 2.18$ \\
Apical & $1.99 \pm 0.92$ & $2.31 \pm 1.00^{\mathrm{b}}$ & & $0.87 \pm 0.06$ & $0.92 \pm 0.04^{\mathrm{b}}$ & & $4.29 \pm 1.67$ & $5.03 \pm 1.74^{\mathrm{b}}$ \\
All & $2.35 \pm 1.21$ & $2.27 \pm 1.02^{\mathrm{a}}$ & & $0.88 \pm 0.06$ & $0.93 \pm 0.03^{\mathrm{a}}$ & & $5.05 \pm 2.18$ & $5.01 \pm 1.84^{\mathrm{a}}$ \\
\hline IOV (All) & $2.20 \pm 1.40$ & $1.74 \pm 1.05$ & & $0.88 \pm 0.08$ & $0.94 \pm 0.04$ & & $5.11 \pm 2.64$ & $4.28 \pm 1.92$ \\
\hline
\end{tabular}

${ }^{\mathrm{a}} p<0.05$, Wilcoxon matched-pairs signed rank test between the automatic and IOV, for all cases.

${ }^{\mathrm{b}} p<0.05$, Mann-Whitney test between automatic and IOV, for each slice pair.

approaches. On average, the tracking approach and estimation required $82 \pm 23 \mathrm{~s}$ per data set (three levels).

The correlation and BA plots are shown in figure 8. In the radial direction, strain was underestimated by the automatic technique compared to the strain obtained through manual delineation. The same biases were observed between observers. Interestingly, the LOAs were narrower for the automatic method compared with the IOV (table 2 and figure 8). In the circumferential direction, although statistically significant biases were found between observers for all levels, the same was not observed for the automatic approach (table 2). Once again, the proposed method showed narrower LOAs when compared with the IOV (table 2 and figure 8). Furthermore, figures 8(c) and (f) give an overview of the strain errors with respect to the segmental level. The largest differences were observed in the lateral segments, with an underestimation of the radial strain and overestimation of the circumferential one.

The difference between the automatic and manual approaches on the entire global strain curve was assessed through the correlation coefficient between each pair of curves. A correlation result of $(0.774,0.989,1.000)\left(5\right.$ th, $50^{\text {th }}$, and 95 th percentiles $)$ and $(0.997,1.000,1.000)$ was achieved for the radial and circumferential strain, respectively, while a correlation of $(0.714,0.999,1.000)$ and $(0.995,1.000,1.000)$ was obtained between observers. The same analysis was performed for the segmental strain curves. The automatic method achieved a correlation of $(0.668,0.986,1.000)$ and $(0.967,0.997,1.000)$ for radial and circumferential 


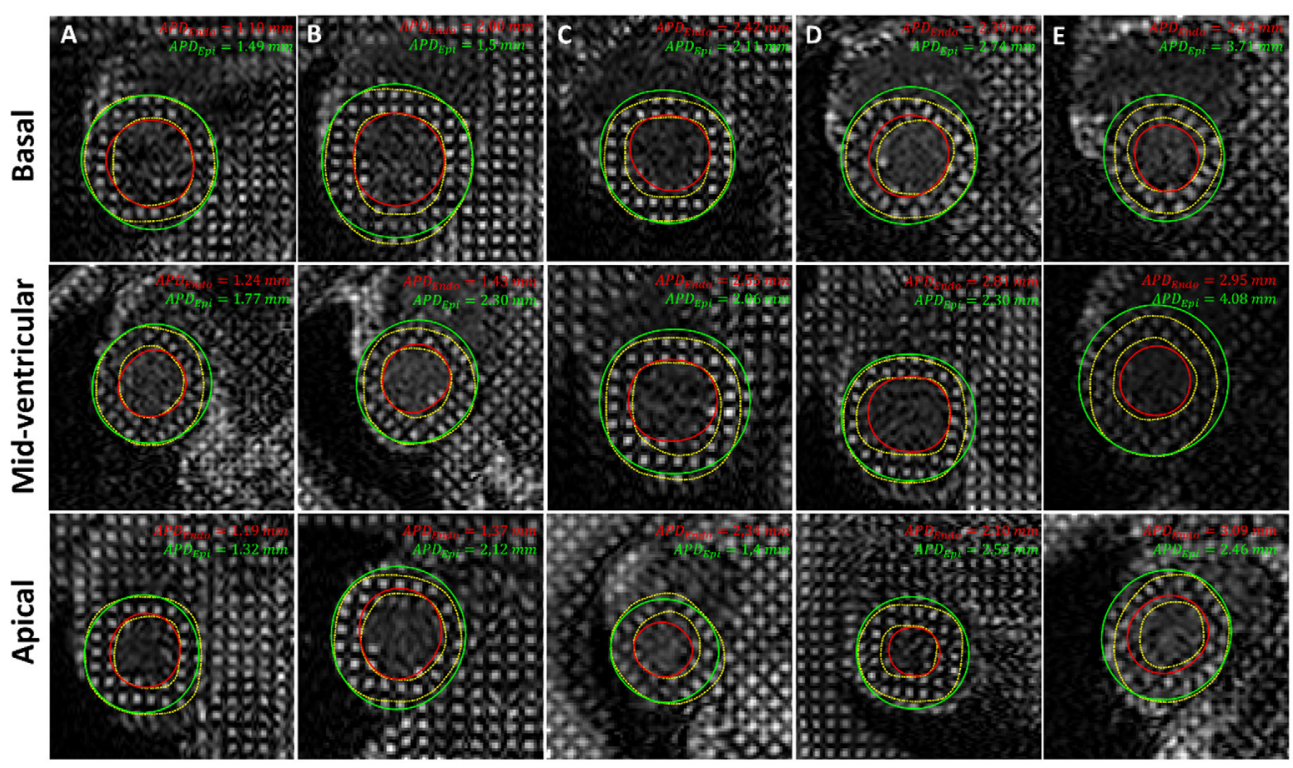

Figure 6. Automatic segmentation results at the 4th frame for the cases corresponding to the (A) 10th, (B) 30th, (C) 50th, (D) 70th, and (E) 90th percentiles, according to the average endo- and epicardial APD (red: endocardium; green: epicardium; yellow: ground truth)

Table 2. Differences of the global and segmental (segm.) radial and circumferential strain value at end-systole using the proposed fully automatic approach or based on an initial manual ROI.

\begin{tabular}{|c|c|c|c|c|c|c|c|}
\hline & & \multicolumn{3}{|c|}{ Radial } & \multicolumn{3}{|c|}{ Circumferential } \\
\hline & & \multirow{2}{*}{$\begin{array}{l}\text { Correlation } \\
\text { coefficient } \\
\text { (R) }\end{array}$} & \multicolumn{2}{|r|}{ BA } & \multirow{2}{*}{$\begin{array}{l}\text { Correlation } \\
\text { coefficient } \\
\text { (R) }\end{array}$} & \multicolumn{2}{|r|}{ BA } \\
\hline & & & $\begin{array}{l}\text { Bias } \\
(\%)\end{array}$ & LOA $(\%)$ & & $\begin{array}{l}\text { Bias } \\
(\%)\end{array}$ & LOA $(\%)$ \\
\hline \multirow[t]{5}{*}{ Global } & Basal & 0.91 & $1.52^{\mathrm{a}}$ & $(-4.18 ; 7.23)$ & 0.97 & $-0.38^{\mathrm{a}}$ & $(-1.72 ; 2.39)$ \\
\hline & Mid & 0.92 & $1.46^{\mathrm{a}}$ & $(-4.15 ; 7.07)$ & 0.95 & 0.01 & $(-2.22 ; 2.24)$ \\
\hline & Apical & 0.88 & $-1.06^{\mathrm{a}}$ & $(-6.69 ; 4.57)^{\mathrm{b}}$ & 0.90 & $0.67^{\mathrm{a}}$ & $(-3.06 ; 4.44)^{b}$ \\
\hline & All & 0.89 & $0.76^{\mathrm{a}}$ & $(-5.30 ; 6.82)^{\mathrm{b}}$ & 0.95 & -0.05 & $(-2.84 ; 2.74)^{\mathrm{b}}$ \\
\hline & IOV (All) & 0.84 & $1.74^{\mathrm{a}}$ & $(-5.82 ; 9.29)$ & 0.93 & $0.60^{\mathrm{a}}$ & $(-2.79 ; 4.00)$ \\
\hline \multirow[t]{2}{*}{ Segm. } & All & 0.92 & $0.96^{\mathrm{a}}$ & $(-9.93 ; 11.85)$ & 0.93 & 0.18 & $(-5.04 ; 5.40)$ \\
\hline & IOV & 0.92 & $1.72^{\mathrm{a}}$ & $(-9.47 ; 12.90)$ & 0.92 & -0.67 & $(-4.96 ; 6.30)$ \\
\hline
\end{tabular}

${ }^{a} p<0.05$, two-tailed paired t-test against zero.

${ }^{\mathrm{b}} p<0.05$, two-tailed F-test between the fully automatic approach (per level and per segment) and its corresponding semi-automatic strategy.

strain, respectively, as opposed to the $(0.650,0.987,1.000)$ and $(0.961,0.997,1.000)$ between observers. No statistical difference was found between approaches.

\subsection{Strain estimated from $t-M R I$ in myocardial segments with different amount of scarring}

Figure 9 relates the strain estimated from t-MRI with scar analysis. A clear relation was found between scar extent and circumferential strain, with statistically significant differences 

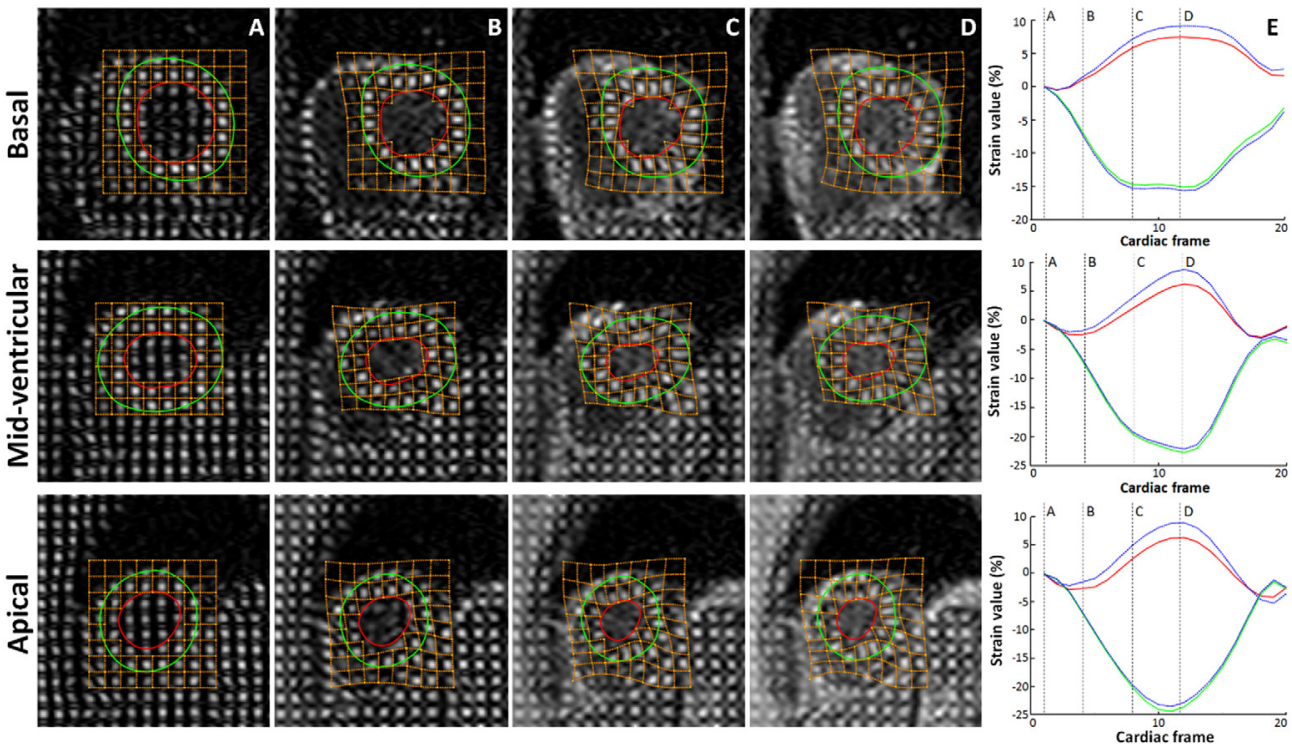

Figure 7. Fully automatic estimation of the global radial and circumferential strain curves for one complete clinical t-MRI data set. A representation of the tracking result (yellow grid) and the contour propagation (red contour - endocardium, green contourepicardium) at (A) end-diastole, (B) and (C) mid-cycle frames, and (D) end-systole. (E) shows the obtained radial (red line) and circumferential (green line) strain curves (the blue lines indicate the result obtained with the manual approach). Extra examples can be found in the multimedia material III (stacks.iop.org/PMB/62/6899/mmedia).
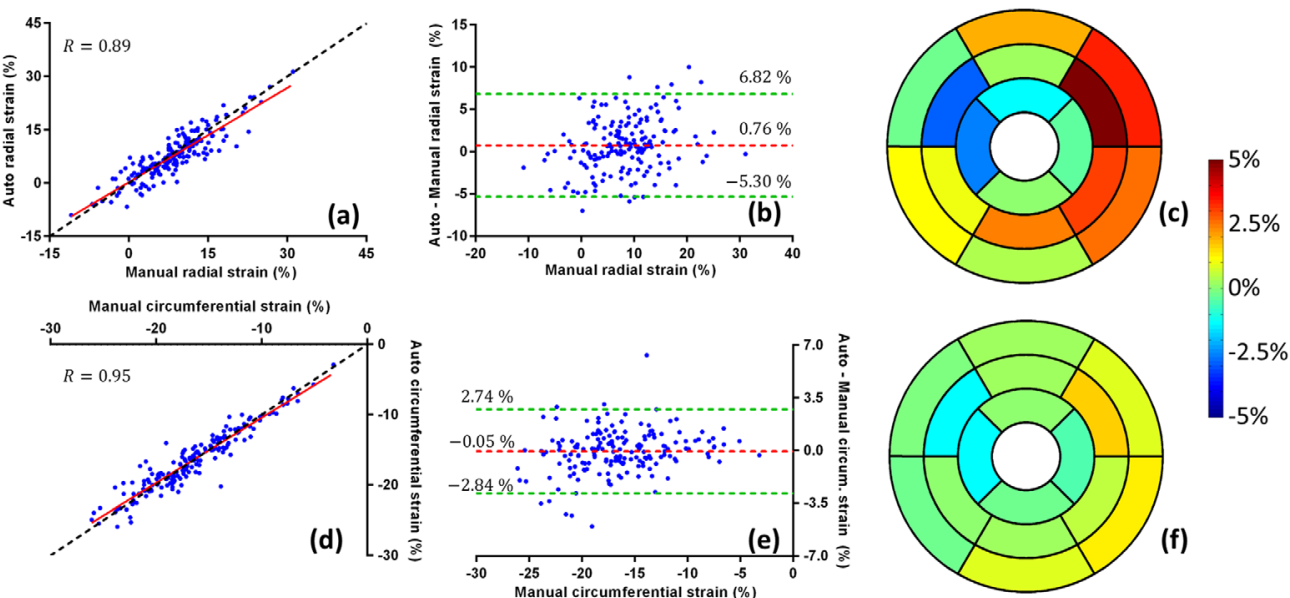

Figure 8. Correlation and BA plots for global ES radial (a) and (b) and circumferential (d) and (e) strain. Mean radial (c) and circumferential (f) strain errors between automatic and manual approaches using the 16 segments model. Dashed lines represent bias (red) and $95 \%$ limits of agreement (green).

between groups. Radial strain showed similar trends, but a clear difference was only seen between normal segments and transmural infarcts. The relation between scar extent and strain values is also illustrated in two different patients (figure 10), showing a correspondence between scar portions and positive and negative circumferential and radial strain, respectively. 


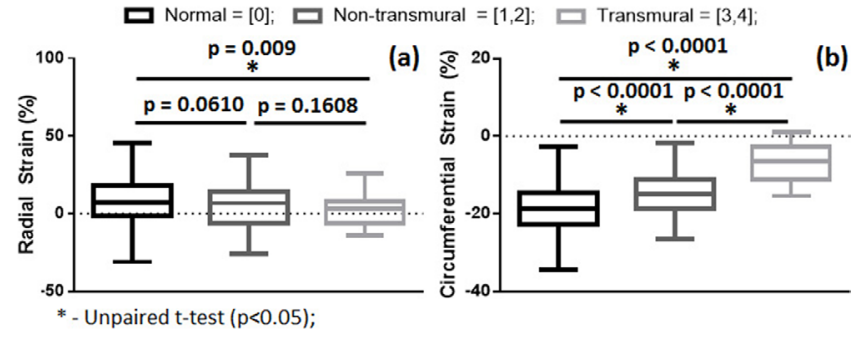

Figure 9. (a) and (b) Segmental radial and circumferential strain versus scar tissue analysis from DE-MRI. $* p<0.05$, unpaired t-test (with Welch's correction) between normal, non-transmural, and transmural regions.

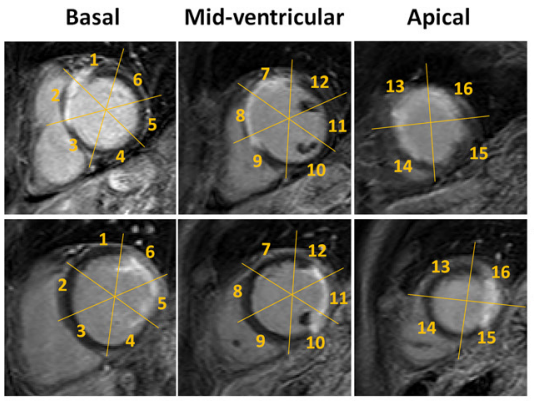

(a) (b) (c)

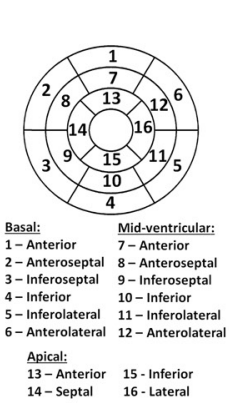

(d)

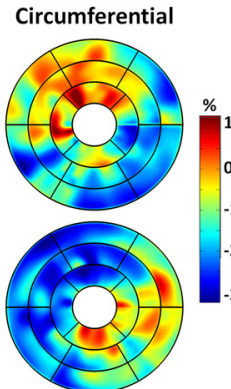

(e)

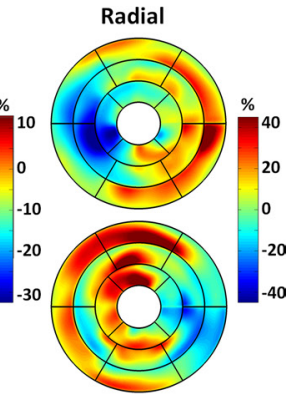

(f)

Figure 10. Illustrative cases of the relationship between scar extent and strain values. (a)-(c) represent the DE-MRI in the basal, mid-ventricular, and apical levels, respectively. (d) describes the 16 segments models and (e) and (f) show the circumferential and radial strain estimated from the t-MRI data set.

\section{Discussion}

A fully automatic framework to estimate strain curves from t-MRI images was proposed. The technique combines a robust automatic-segmentation technique with an image-based tracking approach, allowing the estimation of radial/circumferential strain from t-MRI data sets without any user interaction. Tagged MRI is typically described as the gold standard to extract cardiac strain (Hui and Amini 2012) due to the presence of markers, which facilitate tracking cardiac motion and torsion. Unfortunately, due to its dedicated acquisition and difficulties in identifying the LV, the technique is not widely used in daily practice. As an alternative to reduce the acquisition time, only the most relevant 2D slices (three levels representing the basal, mid-ventricular, and apical region) were acquired. Nevertheless, due to the large interslice distance, focal-abnormal regions may be missed. Recently, a full 3D volume tagging acquisition approach was presented (Chitiboi and Axel 2017). Since the tags are simultaneously generated in all directions, a more realistic and accurate $3 \mathrm{D}$ motion can be estimated from such data sets. However, the acquisition protocol is not straightforward, is recent, and is still being mainly used in the research field (Xu et al 2010, Amano et al 2016, Chitiboi and Axel 2017). In fact, most of the acquisitions are still being performed in animals (Xu et al 2010) or phantom models (Tobon-Gomez et al 2013). Only a small number of cases applied in humans were acquired, requiring thus further studies to prove its added value (Amano et al 2016). Moreover, the 3D tagging reduces the SNR, hampering the motion-estimation stage (Chitiboi and Axel 2017). Another alternative to estimate strain values is strain-encoded MRI. 
In this approach, the myocardial strain is directly extracted from the images during acquisition (Haggerty et al 2014). However, long acquisition times are typically required, and its added value in clinical practice is still being investigated. To date, the strain is therefore typically computed from cine-MRI by using feature tracking techniques. Overall, global strain patterns are similar to those extracted from t-MRI, but cine-MRI typically loses accuracy when assessing regional function (Hor et al 2010, Augustine et al 2013). Given the above, we feel that automating t-MRI analysis remains appealing, as it will reduce processing time and make it attractive for routine practice. The authors would like to mention that the presented framework is currently also being adapted to cine-MRI (multimedia material IV (stacks.iop.org/ $\mathrm{PMB} / 62 / 6899 /$ mmedia)).

More than $95 \%$ of the 204 short-axis slices were successfully segmented using the proposed fully automatic approach. Not only does this illustrate its robustness in realistic data sets but is attractive when compared to manually annotating these data sets due to its low computational time. Note that fewer apical slices were included in the analysis compared to the basal ones, because it was more challenging to obtain manual references for them. Indeed, image quality was generally poorer, and the myocardium smaller compared to the tag dimensions.

The automatic segmentation consists of two consecutive stages: (1) ROI definition and (2) contour initialization and segmentation. Figure 5 illustrates that the detection stage had a high feasibility and accuracy, with median errors already in the same order of magnitude compared to the final segmentation results (see the basal section in figure 5). The highest errors were found when propagating the ROI from one level to another. This can be easily explained by the expected misalignment between slices (Queirós et al 2014). Nevertheless, this initial misalignment is corrected during the subsequent two stages, as presented in figure 5. This further illustrates the robustness of the initialization technique and the need for the two stages at the other levels of the myocardium.

Table 1 presents the segmentation errors obtained by the automatic technique, where the APD and Dice were comparable to those previously presented in cine-MRI (Petitjean and Dacher 2011). No significant differences were obtained between the automatic and IOV for the endocardial region. In contrast, significant differences were found at the epicardial side, consequently showing a better performance of the manual strategy against the automatic method. The low performance at the epicardial region was also previously reported for other automatic MRI segmentation techniques (Petitjean and Dacher 2011, Queirós et al 2015), and can be explained by the low contrast between the epicardium and its neighboring structures. Nonetheless, the obtained epicardial segmentation result still shows a competitive performance, with an APD error much lower than $5 \mathrm{~mm}$ (i.e. threshold typically used in the literature to consider a segmentation successful) and leads to acceptable small differences when assessing strain values. The reader should take into consideration that the performance of the segmentation method is directly related to the spatial resolution of the image. A smaller pixel size (current pixel size is bigger than $1.2 \mathrm{~mm}$ ) increases the details available at the myocardial boundaries, reducing the segmentation errors. However, it also reduces the image SNR and prevents correct motion estimation. Nevertheless, the pixel size should always be smaller than the spacing between tags to allow separation of the tissues and tags.

Assessing the result at the different image levels (table 1), lower errors were globally found at the basal slice, possibly due to the high contrast between tags and tissues in combination with the larger LV wall at this level. Note that although lower APD errors were found at the apical level, this might also be explained by its smaller size, which is confirmed by a lower average Dice. A similar variability in terms of APD error was found between the basal and apical levels, indicating a high flexibility of the proposed methodology to deal with the particularities of the heart. Although the current methodology was only tested on the traditional 
clinical data sets (with three slices), the method also showed potential for acquisitions with more than three slices (see multimedia material V (stacks.iop.org/PMB/62/6899/mmedia)).

Although the current framework was only tested on black blood t-MRI images, this methodology appears to be suitable for white blood acquisitions too. Since the initial LV detection stage (section 2.3.1) searches for heterogeneous regions (i.e. myocardium) and the subsequent LV refinement identifies the tags using a frequency-based strategy (section 2.3.2), an enhancement of the myocardial wall compared to the blood pool is always obtained. As such, after the binarization, the foreground class is always represented by the myocardium, while the background class includes the blood pool (figure 3(b)). Nevertheless, the authors would like to emphasize the assumptions of this segmentation strategy, namely: (1) the LV is roughly centered in the image; (2) no tags (or at least a small number of them) should be detected inside the blood pool; and (3) the myocardial shape is approximately circular or elliptical.

A second experiment was performed to assess the sensitivity of the strain curve with respect to the employed segmentation strategy, i.e. manually or automatically (table 2). Our proposed framework is not only fully automatic but was extensively validated in a large clinical database of t-MRI images. Both aspects have, to the best of our knowledge, not been described elsewhere before. High correlations between automatic and manual approaches were achieved for both global strain components at ES, illustrating the attractiveness of the developed technique for use in clinical practice. Moreover, similar LOAs were found when compared to the IOV ones. This observation indicates that the automatic-segmentation errors are competitive against manual observation during the strain-estimation stage. Furthermore, since the novel method is completely user-independent, the intra- and IOV previously reported in t-MRI validation studies are mitigated (Miller et al 2013, Del-Canto et al 2015). Note that the manual tracking results were evaluated by both observers, and corrected if deemed necessary. Since the current framework is based on an image registration technique, no modification of the tracking result is allowed, being the corrections performed through the modification of the initial manual contour.

When assessing performance per level, it can be observed that at the apical level errors were statistically different, although the LOAs were still narrow (table 2). The former might be related to the magnitude and complexity of the motion, which is typically higher compared to the other levels, as previously reported in Del-Canto et al (2015). Indeed, figure 8 confirms that the performance of the automatic method was lower when motion tended to be larger. The same trends are observed when looking at the spatial error distribution of the strain error (figures 8(c) and (f)). Indeed, the largest strain errors occurred at the lateral wall. Not only is the motion largest there (Del-Canto et al 2015) but the myocardial wall tends to be thinner compared to the other segments (Miller et al 2013). By acquiring a higher number of frames (around 20 frames were used in the current study), the image-registration performance could be improved as fast motion components are finer. However, it is important to note that the frame rate is intrinsically limited by the acquisition protocol.

When comparing both strain components, it is evident that strain curves were retrieved better in the circumferential direction (table 2 and figure 8). This can be explained by the fact that radial strain has to be computed over a small thickness and is therefore sensitive to small variations in the segmentation. The presence (and fading) of the tags over the cardiac cycle may further complicate the radial strain estimation (Tobon-Gomez et al 2013). Indeed, the inferior performance of radial strain is not specific to t-MRI only, having been previously reported for other modalities (Alessandrini et al 2016).

The previous observations were corroborated through the comparison between tagging strain myocardial segments with different amount of scarring estimated from the gold standard (i.e. DE-MRI). This study reinforced the superior performance of circumferential strain over the radial component. Moreover, this analysis is an indicator of the high accuracy of the 
entire framework. Although total separation for the circumferential strain (figure 9) was not observed between the three groups (i.e. normal, non-transmural, and transmural) as previously reported (Koos et al 2013, Nakamura et al 2015), clear differences in terms of median value and strain distribution were observed. Moreover, statistically significant differences were found between the three groups for the circumferential strain. These results indicate that the fully automatic method is sensitive to abnormal regions and is therefore of use in clinical practice to assess pathological cases (see figures 9 and 10).

This study has some limitations, namely: (1) the current segmentation can only be applied on short-axis views; and (2) the automatic segmentation approach was only compared against manual delineations. Comparing our technique to other existing commercial approaches (with semi-automatic segmentation, such as HARP or inTag) (Osman et al 1999, Liu and Prince 2010) would be of interest, but remains unfeasible at this point in time given that the segmentation results could not be directly exported, and therefore not directly compared.

\section{Conclusion}

In the current study, a novel fully automatic framework to estimate strain curves from t-MRI images was proposed. The technique combines a robust and fast automatic-segmentation technique with an image-based tracking approach. It was successfully applied in more than 200 slices with an error comparable to the IOV and allowed the estimation of radial and circumferential strains from the t-MRI data sets without any user interaction and with a performance similar to the traditional practice. Its high feasibility, fully automatic character, and robust estimation of the strain indices makes this solution attractive for use in clinical practice.

\section{Acknowledgments}

The authors acknowledge FCT_-Fundacão para a Ciência e a Tecnologia, Portugal, and the European Social Found, European Union, for funding support through the Programa Operacional Capital Humano (POCH) in the scope of the PhD grants SFRH/BD/95438/2013 (P Morais) and SFRH/BD/93443/2013 (S Queirós). This work was supported by the projects NORTE-07-0124-FEDER-000017 and NORTE-01-0145-FEDER-000013, co-funded by Programa Operacional Regional do Norte, Quadro de Referência Estratégico Nacional, through Fundo Europeu de Desenvolvimento Regional (FEDER). The authors would also like to acknowledge the EU (FP7) framework program, for the financial support of the DOPPLERCIP project (grant no. 223615).

\section{Conflicts of interest}

No conflicts of interest.

\section{References}

Alessandrini M et al 2016 Detailed evaluation of five 3D speckle tracking algorithms using synthetic echocardiographic recordings IEEE Trans. Medical Imaging 35 1915-26

Amano Y, Yamada F, Hashimoto H, Obara M, Asai K and Kumita S 2016 Fast 3-breath-hold 3-dimensional tagging cardiac magnetic resonance in patients with hypertrophic myocardial diseases: a feasibility study Biomed. Res. Int. 20163749489 
Augustine D et al 2013 Global and regional left ventricular myocardial deformation measures by magnetic resonance feature tracking in healthy volunteers: comparison with tagging and relevance of gender J. Cardiovascular Mag. Reson. 151

Axel L, Montillo A and Kim D 2005 Tagged magnetic resonance imaging of the heart: a survey Med. Image Anal. 9 376-93

Barbosa D et al 2013 Fast and fully automatic 3D echocardiographic segmentation using B-spline explicit active surfaces: feasibility study and validation in a clinical setting Ultrasound Med. Biol. 39 89-101

Barbosa D, Dietenbeck T, Schaerer J, D'hooge J, Friboulet D and Bernard O 2012 B-spline explicit active surfaces: An efficient framework for real-time 3D region-based segmentation IEEE Trans. Image Proc. 21 241-51

Cerqueira M D et al 2002 Standardized myocardial segmentation and nomenclature for tomographic imaging of the heart. A statement for healthcare professionals from the Cardiac Imaging Committee of the Council on Clinical Cardiology of the American Heart Association Circulation 105 539-42

Chandrashekara R, Mohiaddin R H and Rueckert D 2004 Analysis of 3D myocardial motion in tagged MR images using nonrigid image registration IEEE Trans. Med. Imaging 23 1245-50

Chitiboi T and Axel L 2017 Magnetic resonance imaging of myocardial strain: a review of current approaches J. Magn. Reson. Imaging (https://doi.org/10.1002/jmri.25718)

Ciofolo C, Fradkin M, Mory B, Hautvast G and Breeuwer M 2008 Automatic myocardium segmentation in late-enhancement MRI 5th IEEE Int. Symp. Biomedical Imaging: from Nano to Macro 2008 (ISBI 2008) pp 225-8

Del-Canto I et al 2015 Characterization of normal regional myocardial function by MRI cardiac tagging J. Magn. Reson. Imaging 41 83-92

Haggerty C M et al 2014 Quantification of left ventricular volumes, mass, and ejection fraction using cine displacement encoding with stimulated echoes (DENSE) MRI J. Mag. Reson. Imaging 40 398-406

Hor K N et al 2010 Comparison of magnetic resonance feature tracking for strain calculation with harmonic phase imaging analysis JACC: Cardiovascular Imaging 3 144-51

Hui W and Amini A A 2012 Cardiac motion and deformation recovery from MRI: a review IEEE Trans. Med. Imaging 31 487-503

Koos R et al 2013 Layer-specific strain-encoded MRI for the evaluation of left ventricular function and infarct transmurality in patients with chronic coronary artery disease Int. J. Cardiol. 166 85-9

Liu X and Prince J L 2010 Shortest path refinement for motion estimation from tagged MR images IEEE Trans. Med. Imaging 29 1560-72

Miller C A et al 2013 Comparison of local sine wave modeling with harmonic phase analysis for the assessment of myocardial strain J. Magn. Reson. Imaging 38 320-8

Morais P, Heyde B, Barbosa D, Queirós S, Claus P and D'hooge J 2013 Cardiac motion and deformation estimation from tagged MRI sequences using a temporal coherent image registration framework Functional Imaging and Modeling of the Heart vol 7945, ed S Ourselin et al (Berlin: Springer) pp 316-24

Nakamura M et al 2015 Quantitative circumferential strain analysis using adenosine triphosphate-stress/ rest $3 \mathrm{~T}$ tagged magnetic resonance to evaluate regional contractile dysfunction in ischemic heart disease Eur. J. Radiol. 84 1493-501

Osman N F, Kerwin W S, McVeigh E R and Prince J L 1999 Cardiac motion tracking using CINE harmonic phase (HARP) magnetic resonance imaging Mag. Reson. Med. 421048

Otsu N 1975 A threshold selection method from gray-level histograms Automatica 11 23-7

Petitjean C and Dacher J-N 2011 A review of segmentation methods in short axis cardiac MR images Med. Image Anal. 15 169-84

Qian Z 2008 Automatic detection, segmentation and motion characterization of the heart from tagged MRI PhD Thesis University of New Jersey

Qian Z, Metaxas D and Axel L 2005 A learning framework for the automatic and accurate segmentation of cardiac tagged MRI images Computer Vision for Biomedical Image Applications vol 3765, ed Y Liu et al (Berlin: Springer) pp 93-102

Queirós S et al 2013 Fast fully automatic segmentation of the myocardium in 2D cine MR images Functional Imaging and Modeling of the Heart (Berlin: Springer) pp 71-9

Queirós S, Barbosa D, Heyde B, Morais P, Vilaça J L, Friboulet D and D'hooge J 2014 Fast automatic myocardial segmentation in 4D cine CMR datasets Med. Image Anal. 18 1115-31

Queirós S et al 2015 Multi-centre validation of an automatic algorithm for fast 4D myocardial segmentation in cine CMR datasets Eur. Heart J. Cardiovasc Imaging 17 1118-27 
Queirós S et al 2016 Aortic valve tract segmentation from 3D-TEE using shape-based B-spline explicit active surfaces IEEE Trans. Med. Imaging 35 2015-25

Rademakers F et al 2013 Determining optimal noninvasive parameters for the prediction of left ventricular remodeling in chronic ischemic patients Scand. Cardiovascular J. 47 329-34

Shi W et al 2012 A comprehensive cardiac motion estimation framework using both untagged and 3D tagged MR images based on nonrigid registration IEEE Trans. Med. Imaging 31 1263-75

Smal I, Carranza-Herrezuelo N, Klein S, Niessen W and Meijering E 2011 Quantitative comparison of tracking methods for motion analysis in tagged MRI IEEE Int. Symp. Biomedical Imaging: From Nano to Macro 2011 pp 345-8

Smiseth O A, Torp H, Opdahl A, Haugaa K H and Urheim S 2015 Myocardial strain imaging: how useful is it in clinical decision making? Eur. Heart J. 37 1196-207

Tobon-Gomez C et al 2013 Benchmarking framework for myocardial tracking and deformation algorithms: an open access database Med. Image Anal. 17 632-48

Townsend N, Nichols M, Scarborough P and Rayner M 2015 Cardiovascular disease in Europeepidemiological update 2015 Eur. Heart J. 36 2696-705

Xu C et al 2010 Deformation analysis of 3D tagged cardiac images using an optical flow method $J$. Cardiovasc Magn. Reson. 12 19-25

Ypenburg C et al 2007 Effect of total scar burden on contrast-enhanced magnetic resonance imaging on response to cardiac resynchronization therapy Am. J. Cardiol. 99 657-60 